\title{
AVALIAÇÃO DA MADEIRA E DA POLPAÇÃO KRAFT EM CLONES DE EUCALIPTOS ${ }^{1}$
}

\author{
Adriana de Fátima Gomes Gouvêa ${ }^{2}$,Paulo Fernando Trugilo ${ }^{3}$, Jorge Luiz Colodette ${ }^{4}$, José Tarcísio \\ Lima $^{3}$, José Reinaldo Moreira da Silva ${ }^{3}$ José Lívio Gomide ${ }^{4}$
}

\begin{abstract}
RESUMO - A produção de celulose de baixo custo e alta qualidade requer madeira adequada e bem selecionada. A seleção de clones superiores tem sido realizada com base em critérios como densidade básica, rendimento gravimétrico da polpação e composição química da madeira, especialmente de celulose, hemiceluloses, extrativos e ligninas. O objetivo deste trabalho foi avaliar as características da madeira de Eucalyptus, por método destrutivo, e a produção de polpa celulósica kraft em seis clones. Utilizaram-se cinco árvores de cada clone, aos 3 anos de idade, plantadas em espaçamento 3,0 m x 3,3 m, nas regiões de Cocais, Guanhães, Rio Doce e Santa Bárbara, Estado de Minas Gerais. A densidade básica foi medida em discos extraídos a 1,3 m de altura do solo (DAP) e em cavacos da árvore inteira (amostra composta). A composição química foi medida em amostras de serragem, retiradas no DAP. Os cozimentos foram efetuados a partir de cavacos da árvore inteira. Verificou-se que a densidade medida no DAP foi ligeiramente superior à medida nos cavacos da árvore toda. A composição química geral da madeira foi muito influenciada pelo tipo de clone, local de plantio e interação. Locais mais montanhosos produziram madeira com maior teor de celulose e menor de hemicelulose. A madeira do clone F da região de Santa Bárbara e Cocais apresentaram madeiras de qualidade inferior para produção de polpa celulósica. Os melhores rendimentos de polpação kraft foram alcançados com o clone B nas regiões de Guanhães e Santa Bárbara.
\end{abstract}

Palavras-chave: Celulose, madeira e Eucalyptus.

\section{WOOD EVALUATION AND KRAFT PULPING IN EUCALYPTS CLONES}

\begin{abstract}
Low cost and high quality cellulose production demands appropriate wood. The selection of superior clones has been done based on some criteria as basic density, gravimetric yield of pulping and wood chemical composition, especially of cellulose, hemicelluloses, extractive and lignin contents. This study aimed at evaluating the characteristics of the Eucalyptus wood, by destructive methods, and the Kraft pulping production in six clones. Five 3-year-old trees of s each clone, planted in a spacing of 3.0 × 3.3 m, were sampled in Cocais, Guanhães, Rio Doce and Santa Bárbara, in Minas Gerais State, Brazil. The basic density was measured in disks collected at $1.3 \mathrm{~m}$ from the ground (DBH) and in whole tree chips (composed sample). The chemical composition was measured in sawdust samples, taken from $D B H$. The pulping was carried out from whole tree chips. It was verified that the density measured in DBH was slightly superior to that measured in the whole tree chips. The general wood chemical composition was very much affected by the planting site and type of clone. Mountain sites produce wood with higher contents of cellulose and lower contents of hemicelluloses. The F clone wood from Santa Bárbara and Cocais presented lower qualities in relation to the other studied clones. The bests kraft pulping yields were achieved from the the clone B from Guanhães and Santa Bárbara.
\end{abstract}

Keywords: Cellulose, wood and Eucalyptus.

\footnotetext{
${ }^{1}$ Recebido em 14.02.2008 e aceito para publicação em 23.06.2009.

${ }^{2}$ Programa de Pós-Graduação em Ciência Florestal da Universidade Federal de Viçosa - Viçosa, MG. E-mail: <agomes@ufv.br>.

${ }^{3}$ Departamento de Engenharia Florestal da Universidade Federal de Lavras (UFLA) - Lavras,MG. E-mail: <trugilho@ufla.br

${ }^{4}$ Departamento de Engenharia Florestal da Universidade Federal de Viçosa - Viçosa,MG. E-mail: <colodett@ufv.br>e <jlgomide@ufv.br>.
} 


\section{INTRODUÇÃO}

A madeira é considerada material desuniforme. A ocorrência das variações pode ser atribuída às espécies, idade, fatores genéticos e ambientais. Dentro dos indivíduos de uma mesma espécie ocorrem diferenças marcantes nas características da madeira com a altura do tronco e no sentido da medula em direção à casca. Além disso, há diferenças significativas entre cerne e alburno, madeira de início e madeira de fim de estação de crescimento. Em escala microscópica, observam-se diferenças até mesmo entre células individuais (BROWNING, 1963). No gênero Eucalyptus, a densidade pode variar com a idade, com o vigor, com o local onde crescem, com a taxa de crescimento e, na mesma árvore, varia no sentido base-topo e na distância medula-casca (SOUZA et al., 1979). As variações no sentido da medula em direção à casca são as mais importantes. A extensão dessa variabilidade é determinada, principalmente, pela presença da madeira juvenil, pela sua proporção relativa no tronco e pelas suas características físicas, químicas e anatômicas. A elevação do gradiente de variação dentro da zona juvenil diminui com o passar do tempo (MALAN, 1995).

A qualidade da madeira é um fator de extrema importância quando o objetivo é a produção de polpa celulósica com alto rendimento industrial, baixo custo e elevada qualidade. Com relação ao processo de polpação, a grande maioria das variáveis já foi bem estudada e, sabe-se, sofre alterações em função da matéria-prima.

Assim, a fim de atingir o objetivo de produção de polpa celulósica de alta qualidade, o maior desafio da indústria é a seleção de matéria-prima adequada para o seu suprimento. Um estudo desenvolvido, com a participação das principais empresas brasileiras produtoras de celulose kraft branqueada de Eucalyptus, para o estabelecimento da qualidade das madeiras dos clones de última geração indicaram alta qualidade dos clones de Eucalyptus atualmente plantados no Brasil para produção de celulose (GOMIDE et al., 2005).

O objetivo deste trabalho foi avaliar as características da madeira, por método destrutivo, e a produção de polpa celulósica kraft em seis clones de eucaliptos, provenientes de quatro locais de plantio.

R. Árvore, Viçosa-MG, v.33, n.6, p.1175-1185, 2009

\section{MATERIAL E MÉTODOS}

\subsection{Material biológico e local de experimentação}

Foram utilizados seis clones de eucalipto sendo (A e E Eucalyptus grandis e B, C, D e E Híbrido de Eucalyptus grandis $x$ Eucalyptus urophilla) em plantios comerciais com espaçamento de 3,0 $\mathrm{m}$ x 3,3 m e 3 anos de idade, provenientes de quatro diferentes locais, no Estado de Minas Gerais. A saber: Cocais, Guanhães, Rio Doce e Santa Bárbara. Foram coletadas 120 árvores, sendo cinco árvores-amostra, por clone e local. Na etapa de polpação kraft foram utilizadas 24 amostras de cavacos, compostas pela mistura das cinco amostrasárvores de cada clone/local.

As árvores foram selecionadas em uma parcela de 10 x 10 plantas, entre as que apresentavam o diâmetro médio \pm um desvio-padrão.

\subsection{Densidade básica da madeira}

A densidade básica da madeira foi determinada por dois métodos de amostragem diferentes: uma determinação a 1,3 m de altura do solo (DAP) e outra com a amostra composta da árvore.

A densidade no DAP foi determinada em duas cunhas opostas retiradas de um disco com $2,5 \mathrm{~cm}$ de espessura, utilizando-se o método da medição indireta do volume (balança hidrostática), descrito pela norma Technical Association of the Pulp and Paper Industry -TAPPI 258 om-85 (2002).

\subsection{Determinação das dimensões das fibras}

A preparação do macerado para a análise das dimensões das fibras foi feita de acordo com o método de Nicholls e Dadswell, descrito por Ramalho (1987). Foram mensuradas 45 fibras por árvore-amostra e por clone e local. As mensurações foram realizadas através do microscópio óptico “Olympus BX 41" acoplado ao sistema de análise de imagem de concepção canadense "Win Cell". As imagens foram coletadas com o auxílio de uma câmera de captura digital para o microcomputador.

\subsection{Determinação da composição química da madeira}

A composição química da madeira foi determinada em serragem obtida em moinho Willey, a partir de amostras retiradas do disco do DAP, conforme a norma TAPPI T 257 om-92 e TAPPI 264 om-88 (Preparation of wood for chemical analysis). Foram realizadas as análises 
químicas a seguir com as seguintes normas: extrativos em acetona (SCAN TEST CM 49-93), lignina insolúvel (GOMIDE e DEMUNER,1986), lignina solúvel (Goldschimid, 1971), grupos acetilas (SOLAR et al., 1987), ácidos urônicos (SCOTT, 1979) e carboidratos (KAAR et al., 1991)

\subsection{Preparo e classificação dos cavacos}

Foram retirados toretes de $1 \mathrm{~m}$ de comprimento na base, 25, 50, 75 e 100\% da altura comercial do tronco, considerado até um diâmetro mínimo de $7 \mathrm{~cm}$. Os toretes foram picados em picador laboratorial na Cenibra. Os cavacos foram classificados manualmente no Laboratório de Celulose e Papel da UFV, sendo selecionados os cavacos retidos em peneira de $16 \mathrm{~mm}$ x $16 \mathrm{~mm}$. Os nós, cunhas e cascas detectados visualmente foram eliminados manualmente no momento da classificação.

\subsection{Polpação kraft}

Nesta etapa, foram utilizadas 24 amostras de cavacos, compostas pela mistura das cinco árvoresamostra de cada clone e local. Os cozimentos kraft foram realizados em digestor rotativo, com quatro reatores individuais de dois litros cada um, aquecido eletricamente e dotado de termômetro e manômetro, possibilitando a realização de quatro cozimentos simultaneamente.

Foi estabelecida uma carga de álcali fixa de $18,4 \%$ para todas as 24 amostras, de acordo com estudos prévio da carga de álcali ativo requerida para kappa 17, como $\mathrm{NaOH}$, que é convencionalmente utilizado pela indústria nacional. O cozimento foi conduzido em $250 \mathrm{~g}$ de cavacos absolutamente secos (a.s.), $25 \%$ de sulfidez, com uma relação licor/madeira de 4/1, na temperatura máxima de $170{ }^{\circ} \mathrm{C}$, tempo na temperatura máxima de 90 min e tempo na temperatura máxima de 60 min. Para a construção dessa curva, foi escolhida a amostra de cavacos proveniente das cinco árvores do clone A da região do rio Doce, por apresentar densidade básica, teor de lignina e extrativos mais próximos da média das 24 amostras.

\subsection{Lavagem e depuração}

Após os cozimentos, os cavacos foram descarregados no lavador de polpa com tela de aço inox de 150 mesh e lavados com água à temperatura ambiente. A individualização das fibras foi realizada em "hidrapulper" laboratorial de 25 litros de capacidade, numa consistência de aproximadamente $0,6 \%$. A depuração da celulose foi realizada em depurador laboratorial Voith dotado de placa com fendas de $0,2 \mathrm{~mm}$. A polpa foi lavada com excesso de água e, posteriormente, desaguada em centrífuga até uma consistência de aproximadamente $30 \%$. Em seguida foram realizadas as análises do número kappa, viscosidade e rendimento depurado.

\subsection{Determinação do número kappa e viscosidade da polpa celulósica}

A formação da folha foi de acordo com a norma TAPPI (2002). As análises de determinação do número kappa e a viscosidade seguiram as normas TAPPI 236 cm-85 e TAPPI 230 om-89, respectivamente.

\subsection{Análise estatística}

Utilizou-se o delineamento inteiramente casualizado disposto em esquema fatorial com dois fatores, clones e locais. Para as características densidade básica, morfologia das fibras e composição química da madeira, foram utilizadas cinco repetições ( 5 árvores-amostra) e para as características de polpação, utilizadas duas repetições (dois cozimentos de amostra composta da madeira das cinco árvores-amostra). De acordo com a significância, procedeu-se ao desdobramento de local dentro de clone, ou seja, o estudo do comportamento dos clones em função de cada local.

\section{RESULTADOS E DISCUSSÃO}

\subsection{Densidade básica e morfologia das fibras}

Na Tabela 1, apresenta-se o resumo da análise de variância do delineamento experimental adotado. Observa-se nessa tabela, que os efeitos de clone e local foram significativos a $1 \%$ de probabilidade para densidade básica da madeira, em cavacos (DBm) e DAP (DBdap), comprimento da fibra (Comp), diâmetro do lume (Lume) e espessura da parede (Espes). O efeito de clones foi não significativo para a largura da fibra (Larg). A interação clone x local foi significativa para DBm, Comp, Lume e Espes, a $1 \%$ de probabilidade, e Larg a 5\% de probabilidade, sendo não significativa para DBdap. Interação significativa indica a existência de dependência entre os fatores. Dessa forma, optou-se por fazer o desdobramento da interação e avaliação do efeito do local (regional) dentro do clone. Observa-se que somente os clones B e F não apresentaram diferença significativa para

R. Árvore, Viçosa-MG, v.33, n.6, p.1175-1185, 2009 
Tabela 1 - Resumo da análise de variância para os resultados de densidade básica da madeira, dimensões das fibras, para o desdobramento da interação e avaliação do efeito de local dentro de clone para densidade básica média e morfologia das fibras.

Table 1- Summary of the wood basic density and fibers dimensions variance analysis and the unfolding interaction and evaluation of the site effect inside the clone for medium basic density and fiber morphology.

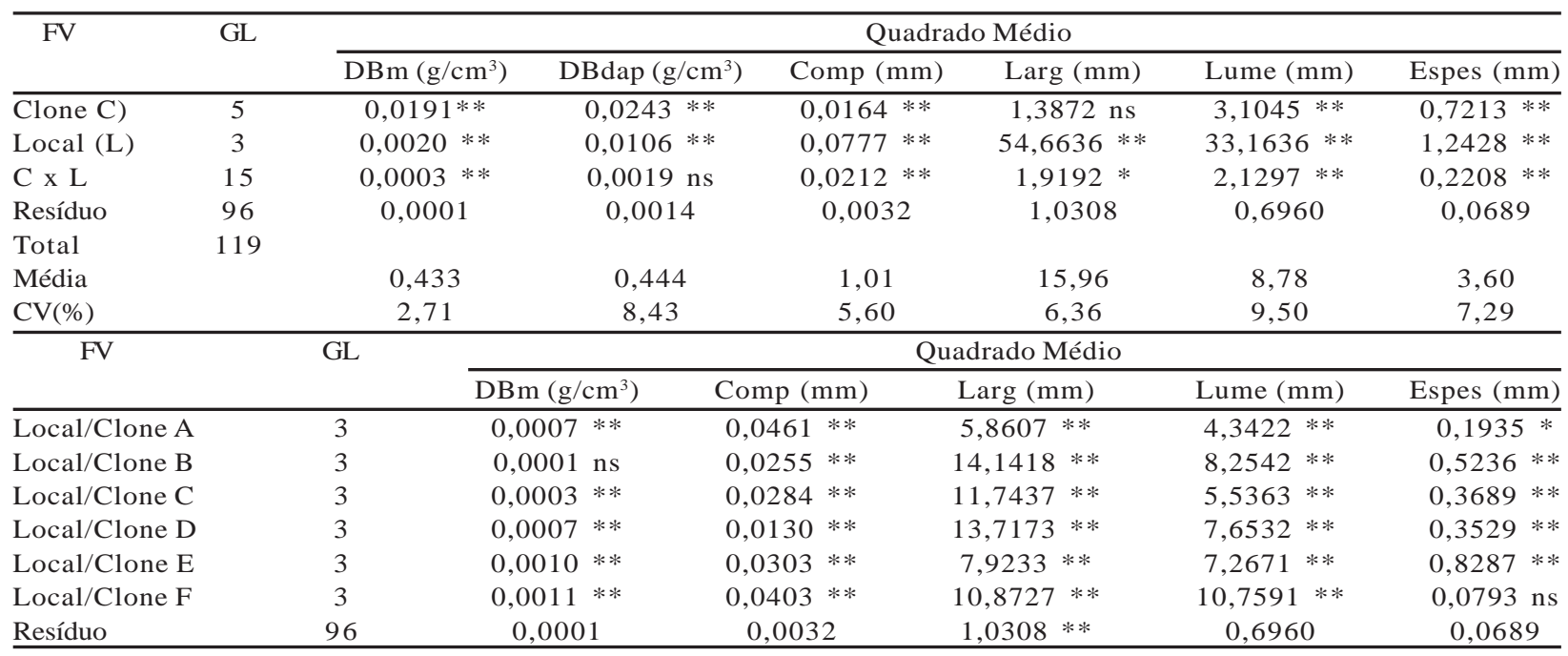

$\mathrm{FV}=$ fonte de variação, GL = grau de liberdade; $* *$, * ns = significativo a 1 e $5 \%$ de probabilidade e não significativo, respectivamente.

DBm e Espes. Para o comprimento, a largura e o diâmetro do lume das fibras, todos os clones apresentaram diferenças nos locais avaliados.

Na Tabela 2, apresentam-se os valores médios e o teste de comparação múltipla para densidade básica (DBm) e a morfologia das fibras. Verifica-se, nessa tabela que na média a densidade básica no DAP foi maior que a dos cavacos obtidos de uma amostra composta de diferentes partes do tronco da árvore, exceto para o clone D. Foi também observada maior variabilidade entre árvores, nas medições efetuadas no DAP. A densidade básica dos cavacos apresentou menor variabilidade entre árvores. Esse fato pode estar relacionado à seleção prévia desses cavacos, sendo usados somente os isentos de defeitos. No entanto, os menores valores médios de densidades básicas observados nos cavacos estão relacionados à variação longitudinal, onde se consideraram frações significativas do tronco que apresentam densidade básica menor que a do DAP. Os clones $\mathrm{C}$ e $\mathrm{F}$ apresentaram o menor e maior valor de densidade básica, respectivamente, em todos os locais avaliados.

R. Árvore, Viçosa-MG, v.33, n.6, p.1175-1185, 2009
Em relação ao comprimento, largura, diâmetro do lume e espessura da parede das fibras, foram verificadas pequenas, porém significativas variações entre clones e locais (Tabela 2). O clone $\mathrm{F}$ apresentou maior diâmetro médio do lume $(9,5 \mathrm{~mm})$, menor espessura média de parede $(3,28 \mathrm{~mm})$ e menor comprimento da fibra em relação aos demais. Esse clone foi o que também apresentou a menor densidade básica no DAP e na média da árvore (cavacos). Esse comportamento está de acordo com alguns pesquisadores (TOMAZELLO FILHO, 1985; FOEKEL et al., 1990), que relataram uma relação positiva entre espessura de parede e densidade básica da madeira.

Em relação aos valores médios de densidade básica por local (Tabela 3), os resultados indicam que os clones das regiões de Cocais e Santa Bárbara apresentaram o maior e menor valor de densidade básica no DAP, contrariamente às medições na árvore inteira que mostram densidade mais alta para Guanhães e mais baixa para Cocais. Esses resultados contraditórios realçam as diferenças nas duas formas de medição da densidade básica, e o ponto de vista industrial, a sua medição na árvore inteira é o mais recomendável. 
Tabela 2-Resultados médios de densidade básica, morfologia de fibras para os clones e locais avaliados. Table 2 - Average results of basic density, fiber morphology for the clones and evaluated sites.

\begin{tabular}{|c|c|c|c|c|c|c|c|}
\hline Clones & Locais & $\mathrm{DBm}$ & DBdap & Comp & Larg & Lume & Espar \\
\hline \multirow[t]{5}{*}{$\mathrm{A}$} & Cocais & $0,418 \mathrm{~b}$ & 0,461 & $1,11 \mathrm{a}$ & $16,32 \mathrm{a}$ & 9,38 a & $3,50 \mathrm{~b}$ \\
\hline & Guanhães & 0,441 a & 0,455 & $0,97 \mathrm{~b}$ & 16,29 a & 8,63 a & 3,86 a \\
\hline & Rio Doce & 0,444 a & 0,455 & $1,04 \mathrm{a}$ & $15,71 \mathrm{a}$ & 8,77 a & $3,49 \mathrm{~b}$ \\
\hline & Sta. Bárbara & $0,434 \mathrm{a}$ & 0,437 & $0,89 \mathrm{c}$ & $14,02 \mathrm{~b}$ & $7,18 \mathrm{~b}$ & $3,42 \mathrm{~b}$ \\
\hline & Média & 0,434 & $0,452 \mathrm{~b}$ & 1,00 & 15,59 & 8,49 & 3,57 \\
\hline \multirow[t]{5}{*}{ B } & Cocais & $0,434 \mathrm{a}$ & 0,472 & $1,10 \mathrm{a}$ & $17,41 \mathrm{a}$ & $9,71 \mathrm{a}$ & $3,88 \quad b$ \\
\hline & Guanhães & $0,439 \mathrm{a}$ & 0,449 & $1,06 \mathrm{a}$ & $17,35 \mathrm{a}$ & 8,86 a & $4,30 \mathrm{a}$ \\
\hline & Rio Doce & 0,429 a & 0,440 & $1,05 \mathrm{a}$ & $16,15 \mathrm{a}$ & 8,78 a & $3,68 \mathrm{~b}$ \\
\hline & Sta. Bárbara & $0,437 \mathrm{a}$ & 0,429 & $0,93 \mathrm{~b}$ & $13,81 \mathrm{~b}$ & $6,69 \mathrm{~b}$ & $3,56 \mathrm{~b}$ \\
\hline & Média & 0,435 & $0,448 \mathrm{~b}$ & 1,04 & 16,18 & 8,51 & 3,86 \\
\hline \multirow[t]{5}{*}{$\mathrm{C}$} & Cocais & $0,453 \mathrm{~b}$ & 0,511 & $1,02 \mathrm{~b}$ & $17,73 \mathrm{a}$ & 9,92 a & $3,93 \mathrm{a}$ \\
\hline & Guanhães & $0,467 \mathrm{a}$ & 0,460 & $1,11 \mathrm{a}$ & $16,88 \mathrm{a}$ & 9,46 a & $3,73 \mathrm{a}$ \\
\hline & Rio Doce & $0,471 \mathrm{~b}$ & 0,464 & $1,07 \mathrm{a}$ & $16,40 \mathrm{a}$ & 8,74 a & $3,84 \mathrm{a}$ \\
\hline & Sta Bárbara & 0,456 a & 0,446 & $0,94 \mathrm{c}$ & $14,14 \mathrm{~b}$ & $7,51 \mathrm{~b}$ & $3,31 \mathrm{~b}$ \\
\hline & Média & 0,462 & $0,470 \mathrm{a}$ & 1,04 & 16,29 & 8,91 & 3,70 \\
\hline \multirow[t]{5}{*}{$\mathrm{D}$} & Cocais & $0,430 \mathrm{~b}$ & 0,422 & $1,05 \mathrm{a}$ & $17,50 \mathrm{a}$ & $10,27 \mathrm{a}$ & $3,65 \mathrm{a}$ \\
\hline & Guanhães & 0,459 a & 0,450 & $1,06 \mathrm{a}$ & $17,06 \mathrm{a}$ & $9,40 \mathrm{a}$ & 3,86 a \\
\hline & Rio Doce & $0,447 \mathrm{a}$ & 0,439 & $1,00 \mathrm{~b}$ & $14,69 \mathrm{~b}$ & $8,05 \mathrm{~b}$ & $3,32 \mathrm{~b}$ \\
\hline & Sta. Bárbara & 0,446 a & 0,438 & $0,95 \mathrm{~b}$ & $14,21 \mathrm{~b}$ & $7,57 \mathrm{~b}$ & $3,32 \mathrm{~b}$ \\
\hline & Média & 0,446 & $0,437 \mathrm{~b}$ & 1,02 & 15,87 & 8,82 & 3,54 \\
\hline \multirow[t]{5}{*}{$\mathrm{E}$} & Cocais & $0,439 \mathrm{~b}$ & 0,545 & $1,07 \mathrm{a}$ & $16,85 \mathrm{a}$ & $10,13 \mathrm{a}$ & $3,40 \mathrm{~b}$ \\
\hline & Guanhães & $0,464 \mathrm{a}$ & 0,461 & $1,10 \mathrm{a}$ & $16,76 \mathrm{a}$ & $8,30 \mathrm{~b}$ & $4,27 \mathrm{a}$ \\
\hline & Rio Doce & $0,451 \mathrm{a}$ & 0,459 & $0,93 \mathrm{~b}$ & $15,24 \mathrm{~b}$ & $8,29 \mathrm{~b}$ & $3,48 \mathrm{~b}$ \\
\hline & Sta. Bárbara & $0,431 \mathrm{~b}$ & 0,427 & $0,97 \mathrm{~b}$ & $14,24 \mathrm{~b}$ & $7,22 \mathrm{c}$ & $3,51 \mathrm{~b}$ \\
\hline & Média & 0,446 & $0,473 \mathrm{a}$ & 1,02 & 15,77 & 8,49 & 3,67 \\
\hline \multirow[t]{5}{*}{$\mathrm{F}$} & Cocais & $0,372 \mathrm{~b}$ & 0,379 & $0,90 \mathrm{~b}$ & $16,70 \mathrm{a}$ & $9,84 \mathrm{~b}$ & $3,47 \mathrm{a}$ \\
\hline & Guanhães & $0,372 \mathrm{~b}$ & 0,375 & $1,07 \mathrm{a}$ & $17,76 \mathrm{a}$ & $11,40 \mathrm{a}$ & $3,21 \mathrm{a}$ \\
\hline & Rio Doce & $0,392 \mathrm{a}$ & 0,396 & $0,87 \mathrm{~b}$ & $14,44 \mathrm{~b}$ & $8,05 \mathrm{c}$ & $3,20 \mathrm{a}$ \\
\hline & Sta. Bárbara & $0,355 \mathrm{c}$ & 0,357 & $1,00 \mathrm{a}$ & $15,27 \mathrm{~b}$ & $8,70 \mathrm{c}$ & $3,29 \mathrm{a}$ \\
\hline & Média & 0,373 & $0,377 \mathrm{c}$ & 0,96 & 16,04 & 9,50 & 3,29 \\
\hline
\end{tabular}

DBdap e DBm = densidade básica a 1,3 m de altura do solo (DAP) e densidade básica média dos cavacos $\left(\mathrm{g} / \mathrm{cm}^{3}\right), \mathrm{Comp}$, Larg, Lume e Espar = comprimento da fibra $(\mathrm{mm})$, largura da fibra $(\mathrm{mm})$, diâmetro do lume $(\mathrm{mm})$ e espessura da parede $(\mathrm{mm})$. Médias seguidas de mesma letra, dentro de clone, não diferem entre si pelo teste de Scott-Knott a $5 \%$ de probabilidade.

De modo geral, a espessura da parede da fibra foi menor na regional de Santa Bárbara, exceto para o clone E.

\subsection{Composição química da madeira}

Na Tabela 4, apresenta-se o resumo da análise de variância das características químicas da madeira e o desdobramento do efeito do local dentro do clone. Verifica-se que as interações clone x local foram significativas a $1 \%$ de probabilidade. Quanto aos teores de celulose e de hemiceluloses, todos os clones apresentaram diferenças significativas nos locais de plantios, enquanto no teor de lignina total os clones A, C, D e F não sofreram variação significativa devido aos locais de plantios. Com relação ao teor de extrativos, os clones A, C, D e E não sofreram influência significativa do local de plantio.

Nas Tabelas 5 e 6, apresentam-se os valores médios das características químicas das madeiras estudadas, por clone e por região. Os clones plantados em regiões montanhosas, como os clones A, C e D em Cocais e o F em Rio Doce, produziram madeira com maior teor de celulose. Os resultados da Tabela 6 indicam que as madeiras da região de Cocais se destacaram pelos seus maiores teores de celulose, com exceção do clone E, sendo estatisticamente diferentes das demais. Esse resultado é explicado pelo fato de as árvores de regiões montanhosas possuírem, usualmente, maior quantidade

R. Árvore, Viçosa-MG, v.33, n.6, p.1175-1185, 2009 
Tabela 3 - Valores médios dos efeitos principais para a densidade básica obtida na altura do DAP.

Table 3 - Average values of the main effects for the basic density obtained in the $\mathrm{DBH}$.

\begin{tabular}{lcc}
\hline \multicolumn{1}{c}{ Local } & $\mathrm{DBm}\left(\mathrm{g} / \mathrm{cm}^{3}\right)$ & $\mathrm{DBdap}\left(\mathrm{g} / \mathrm{cm}^{3}\right)$ \\
\hline Cocais & $0,424 \mathrm{~b}$ & $0,468 \mathrm{a}$ \\
Guanhães & $0,440 \mathrm{a}$ & $0,442 \mathrm{~b}$ \\
Rio Doce & $0,439 \mathrm{a}$ & $0,442 \mathrm{~b}$ \\
Santa Bárbara & $0,426 \mathrm{~b}$ & $0,422 \mathrm{c}$ \\
\hline
\end{tabular}

de madeira de tração, que contêm a camada gelatinosa na parede celular, que é rica em celulose (TIMELL, 1969).

Os clones E e F apresentaram os maiores teores de hemiceluloses, respectivamente de $27,4 \%$ e $27,5 \%$. Os menores valores desses polissacarídeos foram observados nos clones A $(25,2 \%)$ e C $(25,8 \%)$. O teor de extrativos das madeiras variou de $0,70 \%$ a $1,71 \%$, sendo estatisticamente não significativas as diferenças entre os vários clones e locais, exceto para o clone D da região de Santa Bárbara, que apresentou teor de extrativo significativamente inferior ao das demais regiões (Tabela 5).

O teor de lignina variou de $27,9 \%$ a $32,2 \%$. As madeiras com maiores teores de lignina estão nas regiões do Rio Doce e Santa Bárbara (Tabela 5), especialmente nos clones A e F.
De acordo com Wallis et al. (1996), um bom clone a ser destinado à produção de celulose e papel é aquele que apresenta alto teor de celulose. Pelos resultados, observou-se que o clone F da região de Santa Bárbara foi o que apresentou a pior qualidade da madeira para a produção de polpa celulósica. Esse clone apresentou atributos como o alto teor de lignina $(32,2 \%)$, baixo teor de celulose $(38,7 \%)$ e alto teor de extrativos $(1,34 \%)$, em comparação com os demais. Além disso, esse clone foi o que apresentou a menor densidade básica, entre todos (Tabela 2). A densidade básica da madeira tem sido considerada muito importantes para o desempenho da polpação kraft (GOMIDE et al., 2005). Além disso, o teor de hemiceluloses da madeira, por dar origem aos chamados ácidos hexenurônicos, tem sido considerado relevante durante a polpação kraft (COSTA et al., 2001), e os extrativos causam prejuízos também por dificultarem a impregnação de cavacos (WEHR e BARRICHELO, 1992).

\subsection{Polpação kraft}

Na Tabela 7, apresenta-se o resumo da análise de variância das características da polpação da madeira. Observa-se, nessa tabela, que os efeitos de clone, local e interação clone x local foram significativos para todas as características avaliadas.

Tabela 4 - Resumo da análise de variância para os resultados das análises químicas da madeira e para o desdobramento da interação e avaliação do efeito de local dentro de clones para as análises químicas da madeira.

Table 4 - Summary of the variance analysis of the wood chemical analyses and the unfolding interaction and evaluation te effect inside the clones for the wood chemical analyses.

\begin{tabular}{|c|c|c|c|c|c|}
\hline \multirow[t]{2}{*}{ FV } & \multirow[t]{2}{*}{ GL } & \multicolumn{4}{|c|}{ Quadrado Médio } \\
\hline & & Celulose (\%) & Hemiceluloses (\%) & Lignina Total $(\%)$ & Extrativos $(\%)$ \\
\hline$\overline{\text { Clone (C) }}$ & 5 & $34,9081 * *$ & $15,7815 * *$ & $13,1403 * *$ & $0,1827 \mathrm{~ns}$ \\
\hline Local (L) & 3 & $26,3317 * *$ & $12,9064 * *$ & $6,2266 * *$ & $0,2063 \mathrm{~ns}$ \\
\hline $\mathrm{CxL}$ & 15 & $15,1442 * *$ & $5,9534 * *$ & $3,8336 * *$ & $0,3973 * *$ \\
\hline Resíduo & 96 & 2,0224 & 0,6860 & 1,1910 & 0,1307 \\
\hline Total & 119 & & & & \\
\hline Média & & 41,94 & 26,50 & 30,46 & 1,1054 \\
\hline $\mathrm{CV}(\%)$ & & 3,39 & 3,13 & 3,58 & 32,71 \\
\hline \multirow[t]{2}{*}{$\mathrm{FV}$} & GL & \multicolumn{4}{|c|}{ Quadrado Médio } \\
\hline & & Celulose $(\%)$ & Hemiceluloses (\%) & Lignina Total $(\%)$ & Extrativos $(\%)$ \\
\hline Local/Clone A & 3 & $24,8395 * *$ & $16,8589 * *$ & $1,5654 \mathrm{~ns}$ & $0,1108 \mathrm{~ns}$ \\
\hline Local/Clone B & 3 & $11,8722 * *$ & $5,4424 * *$ & $5,9419 * *$ & $0,2863 \mathrm{~ns}$ \\
\hline Local/Clone C & 3 & $27,2512 * *$ & $10,3625 * *$ & $2,6049 \mathrm{~ns}$ & $0,3574 *$ \\
\hline Local/Clone D & 3 & $12,9698 * *$ & $5,0065 * *$ & $2,7257 \mathrm{~ns}$ & $1,0591 * *$ \\
\hline Local/Clone E & 3 & $19,6628 * *$ & $2,4342 *$ & $11,2945 * *$ & $0,2956 \mathrm{~ns}$ \\
\hline Local/Clone F & 3 & $5,4574 *$ & $2,5689 *$ & $1,2621 \mathrm{~ns}$ & $0,0834 \mathrm{~ns}$ \\
\hline Resíduo & 96 & 2,0224 & 0,6860 & 1,1910 & 0,1307 \\
\hline
\end{tabular}

$\mathrm{FV}=$ fonte de variação, GL = grau de liberdade; $* *$, * ns = significativo a 1 e $5 \%$ de probabilidade e não significativo, respectivamente. 
Tabela 5-Valores médios de características químicas da madeira dos clones nos locais avaliados.

Table 5 - Average results of clones wood chemical characteristics in the evaluated sites.

\begin{tabular}{|c|c|c|c|c|c|}
\hline Clones & Locais & Celulose* & Hemiceluloses** & Ligninas*** & Extrativos**** \\
\hline \multirow[t]{5}{*}{ A } & Cocais & $45,50 \mathrm{a}$ & $22,74 \mathrm{c}$ & $30,80 \mathrm{a}$ & 0,96 a \\
\hline & Guanhães & $42,25 \mathrm{~b}$ & $26,26 \mathrm{a}$ & $30,56 \mathrm{a}$ & $0,93 \mathrm{a}$ \\
\hline & Rio Doce & $42,50 \mathrm{~b}$ & $25,05 \mathrm{~b}$ & 31,22 a & $1,22 \mathrm{a}$ \\
\hline & Sta. Bárbara & $40,08 \mathrm{c}$ & $26,91 \mathrm{a}$ & 31,83 a & $1,18 \mathrm{a}$ \\
\hline & Média & $42,58 \mathrm{a}$ & $25,24 \mathrm{~d}$ & $31,10 \mathrm{a}$ & $1,07 \mathrm{a}$ \\
\hline \multirow[t]{5}{*}{ B } & Cocais & $44,15 \mathrm{a}$ & $25,44 \mathrm{~b}$ & $29,70 \quad b$ & $0,71 \mathrm{a}$ \\
\hline & Guanhães & 44,78 a & $25,71 \mathrm{~b}$ & $28,58 \mathrm{~b}$ & 0,92 a \\
\hline & Rio Doce & $41,54 \mathrm{~b}$ & $26,14 \mathrm{~b}$ & $30,98 \mathrm{a}$ & $1,34 \mathrm{a}$ \\
\hline & Sta. Bárbara & $42,22 \mathrm{~b}$ & 27,77 a & $28,81 \mathrm{~b}$ & $1,20 \mathrm{a}$ \\
\hline & Média & $43,17 \mathrm{a}$ & $26,27 \mathrm{c}$ & $29,52 \mathrm{~b}$ & $1,04 \mathrm{a}$ \\
\hline \multirow[t]{5}{*}{$\mathrm{C}$} & Cocais & $45,96 \mathrm{a}$ & $24,36 \mathrm{c}$ & 28,92 a & 0,77 a \\
\hline & Guanhães & $40,26 \mathrm{c}$ & 27,68 a & 30,48 a & $1,57 \mathrm{a}$ \\
\hline & Rio Doce & $43,54 \mathrm{~b}$ & $25,12 \mathrm{c}$ & 30,26 a & $1,08 \mathrm{a}$ \\
\hline & Sta. Bárbara & $43,06 \mathrm{~b}$ & $26,17 \mathrm{~b}$ & 29,48 a & $1,29 \mathrm{a}$ \\
\hline & Média & $43,21 \mathrm{a}$ & $25,83 \mathrm{c}$ & $29,79 \mathrm{~b}$ & $1,18 \mathrm{a}$ \\
\hline \multirow[t]{5}{*}{$\mathrm{D}$} & Cocais & $43,71 \mathrm{a}$ & $25,47 \mathrm{~b}$ & 29,97 a & $0,85 \mathrm{a}$ \\
\hline & Guanhães & $40,29 \mathrm{~b}$ & $27,63 \mathrm{a}$ & 30,36 a & $1,71 \mathrm{a}$ \\
\hline & Rio Doce & $40,31 \mathrm{~b}$ & 27,48 a & 30,88 a & $1,32 \mathrm{a}$ \\
\hline & Sta. Bárbara & $41,13 \mathrm{~b}$ & $26,48 \mathrm{~b}$ & $31,68 \mathrm{a}$ & $0,70 \mathrm{~b}$ \\
\hline & Média & $41,36 \mathrm{a}$ & $26,77 \mathrm{~b}$ & $30,72 \mathrm{a}$ & $1,15 \mathrm{a}$ \\
\hline \multirow[t]{5}{*}{$\mathrm{E}$} & Cocais & $40,15 \mathrm{~b}$ & $27,81 \mathrm{a}$ & $30,70 \mathrm{a}$ & $1,35 \mathrm{a}$ \\
\hline & Guanhães & $44,51 \mathrm{a}$ & $26,59 \mathrm{~b}$ & $27,91 \mathrm{~b}$ & 0,99 a \\
\hline & Rio Doce & $40,91 \mathrm{~b}$ & $28,10 \mathrm{a}$ & 30,19 a & $0,80 \mathrm{a}$ \\
\hline & Sta. Bárbara & $40,73 \mathrm{~b}$ & $27,01 \mathrm{~b}$ & $31,38 \mathrm{a}$ & $0,87 \mathrm{a}$ \\
\hline & Média & $41,58 \mathrm{a}$ & 27,38 a & $30,05 \mathrm{~b}$ & $1,00 \mathrm{a}$ \\
\hline \multirow[t]{5}{*}{$\mathrm{F}$} & Cocais & $39,90 \mathrm{a}$ & $27,87 \mathrm{a}$ & $31,10 \mathrm{a}$ & $1,13 \mathrm{a}$ \\
\hline & Guanhães & $39,28 \mathrm{a}$ & $27,85 \mathrm{a}$ & 31,84 a & 1,03 a \\
\hline & Rio Doce & 41,16 a & $26,43 \mathrm{~b}$ & 31,26 a & $1,15 \mathrm{a}$ \\
\hline & Sta. Bárbara & 38,74 a & $27,88 \mathrm{a}$ & $32,18 \mathrm{a}$ & $1,34 \mathrm{a}$ \\
\hline & Média & $39,77 \mathrm{c}$ & $27,51 \mathrm{a}$ & $31,60 \mathrm{a}$ & $1,16 \mathrm{a}$ \\
\hline
\end{tabular}

Médias seguidas de mesma letra, dentro de clone, não diferem entre si pelo teste de Scott-Knott a 5\% de probabilidade. * Para o cálculo do teor de celulose, foi utilizado o teor de glicose total, descontado do teor de glicose associado às glicomananas ** Somatório das hemiceluloses de xilanas, mananas, arabinanas, galactanas, ácidos urôncios e acetil *** Somatório de lignina insolúvel e solúvel **** Extrativos solúveis em acetona.

Tabela 6 - Composição química da madeira por regional.

Table 6 - Chemical composition of the wood by site.

\begin{tabular}{lcccc}
\hline \multicolumn{1}{c}{ Local } & Celulose $(\%)$ & Hemiceluloses $(\%)$ & Lignina Total $(\%)$ & Extrativos $(\%)$ \\
\hline Cocais & $43,23 \mathrm{a}$ & $25,61 \mathrm{c}$ & $30,19 \mathrm{~b}$ & $0,99 \mathrm{a}$ \\
Guanhães & $41,89 \mathrm{~b}$ & $26,95 \mathrm{a}$ & $29,96 \mathrm{~b}$ & $1,19 \mathrm{a}$ \\
Rio Doce & $41,66 \mathrm{~b}$ & $26,38 \mathrm{~b}$ & $30,79 \mathrm{a}$ & $1,12 \mathrm{a}$ \\
Santa Bárbara & $40,99 \mathrm{c}$ & $27,03 \mathrm{a}$ & $30,89 \mathrm{a}$ & $1,09 \mathrm{a}$ \\
\hline
\end{tabular}

Devido à significância da interação, procedeu-se ao seu desdobramento e avaliação do efeito do local dentro do clone, como mostrado na Tabela 7. Por essa tabela, pode-se observar que somente os clones A e C não apresentaram diferenças significativas no número kappa nas quatro regionais avaliadas. Para o rendimento depurado, os clones A e D não apresentaram diferenças significativas nas regionais, respectivamente. Em relação à viscosidade, todos os clones apresentaram diferenças significativas nas 
Tabela 7 - Resumo da análise de variância para características do cozimento e para desdobramento da interação e avaliação do efeito de local dentro de clone para os resultados de cozimento.

Table 7 - Summary of the variance analysis for pulping characteristics and unfolding interaction and evaluation of the site effect inside the clone for the pulping results.

\begin{tabular}{|c|c|c|c|c|}
\hline \multirow[t]{2}{*}{ FV } & \multirow[t]{2}{*}{ GL } & \multicolumn{3}{|c|}{ Quadrado Médio } \\
\hline & & kappa & $\mathrm{RD}$ & Viscosidade \\
\hline Clone (C) & 3 & $1,0000 * *$ & $0,3557 *$ & $47,3255 * *$ \\
\hline Local (L) & 5 & $2,5833 * *$ & $1,4978 * *$ & $136,174 * *$ \\
\hline $\mathrm{C} \times \mathrm{L}$ & 15 & $0,8500 * *$ & $0,8910 * *$ & $17,7495 * *$ \\
\hline Resíduo & 24 & 0,1666 & 0,1043 & 0,7308 \\
\hline Total & 47 & & & \\
\hline Média & & 16,33 & 51,39 & 35,17 \\
\hline $\mathrm{CV}(\%)$ & & 2,50 & 0,63 & 2,43 \\
\hline \multirow[t]{2}{*}{$\mathrm{FV}$} & GL & \multicolumn{3}{|c|}{ Quadrado Médio } \\
\hline & & kappa & $\mathrm{RD}$ & Viscosidade \\
\hline Local/Clone A & 3 & $0,3333 \mathrm{~ns}$ & $0,0012 \mathrm{~ns}$ & $16,1250 * *$ \\
\hline Local/Clone B & 3 & $1,8333 * *$ & $2,1045 * *$ & $60,3945 * *$ \\
\hline Local/Clone C & 3 & $0,4583 \mathrm{~ns}$ & $0,5245 * *$ & $3,6133 * *$ \\
\hline Local/Clone D & 3 & $0,6666 *$ & $0,1345 \mathrm{~ns}$ & $12,0445 * *$ \\
\hline Local/Clone E & 3 & $1,1250 * *$ & $0,4816 *$ & $37,8945 * *$ \\
\hline Local/Clone F & 3 & $0,8333 * *$ & $1,5645 * *$ & $6,0012 * *$ \\
\hline Resíduo & 24 & 0,1666 & 0,1043 & 0,7308 \\
\hline
\end{tabular}

$\mathrm{FV}=$ fonte de variação, $\mathrm{GL}=$ grau de liberdade; $* *, *$ e $\mathrm{ns}=$ significativo a 1 e $5 \%$ de probabilidade e não significativo, respectivamente.

regionais a $1 \%$ de probabilidade. A viscosidade da polpa celulósica foi a característica mais afetada pela regional avaliada. O clone $\mathrm{A}$ foi o clone mais estável nas regionais, exceto para a viscosidade da polpa. Os clones B e F foram os que apresentaram diferenças significativas em todas as características e em todas as regionais, sendo considerados menos estáveis e muito influenciados pelo ambiente onde são plantados.

Os valores médios das características obtidas nos cozimentos kraft estão apresentados na Tabela 8. A taxa de deslignificação, medida pelo número kappa após cozimento, variou de 14,6 (clone C da regional Santa Bárbara) a 17,5 (clone A da regional Cocais) para uma carga de álcali ativo fixa de $18,4 \%$, como $\mathrm{NaOH}$. O número kappa está relacionado com o teor de lignina residual na polpa produzida, ou seja, quanto maior o kappa, maior a quantidade de lignina residual na polpa e mais difícil será o seu branqueamento. O número kappa da polpa indica também a polpabilidade da madeira, já que o álcali ativo foi constante todas as 24 amostras. Pelos valores de número kappa, o clone $\mathrm{C}$ foi o que apresentou a madeira mais fácil de ser cozinhada. A polpabilidade da madeira produzida na regional de Santa Bárbara foi significativamente superior à das demais regiões (Tabela 9).

O rendimento depurado (RD) do cozimento variou de $50,3 \%$ a $52,9 \%$, sendo mais alto para o clone B na regional de Guanhães e mais baixo para o clone $\mathrm{F}$ da regional Cocais. Menores rendimentos depurados foram obtidos nas regionais de Cocais e Rio Doce (Tabela 9).

A viscosidade está relacionada com a degradação dos carboidratos provocada pelos reagentes de cozimento. Ela dá ideia da seletividade do cozimento kraft. Maiores valores de viscosidade indicam melhor seletividade. Neste estudo, foram obtidas viscosidades variando de $26,0 \mathrm{cP}$ a 44,3 cP, indicando significativa variação entre as madeiras. Os maiores valores de viscosidade da polpa marrom foram alcançados com a madeira do clone $\mathrm{B}$, regional do Rio Doce, enquanto a de menor viscosidade foi obtida com a madeira do clone $\mathrm{C}$, na mesma regional. Maiores seletividades de cozimento foram observadas nas regiões de Guanhães e Santa Bárbara (Tabela 9). 
Tabela 8-Valores médios experimentais do cozimento kraft dos clones nos locais avaliados.

Table 8 - Experimental average values of the clone Kraft pulping in the evaluated sites.

\begin{tabular}{|c|c|c|c|c|}
\hline \multirow[t]{2}{*}{ Clone } & \multirow[t]{2}{*}{ Local } & \multicolumn{3}{|c|}{ CARACTERÍSTICA } \\
\hline & & Kappa & $\mathrm{RD}(\%)$ & Viscosidade (cP) \\
\hline \multirow[t]{5}{*}{ A } & Cocais & $17,5 \mathrm{a}$ & $51,8 \mathrm{a}$ & $31,0 \mathrm{~b}$ \\
\hline & Guanhães & $17,3 \mathrm{a}$ & 51,8 a & 36,6 a \\
\hline & Rio Doce & $16,5 \mathrm{a}$ & $51,8 \mathrm{a}$ & $32,3 \mathrm{~b}$ \\
\hline & Santa Bárbara & $17,2 \mathrm{a}$ & 51,9 a & 35,8 a \\
\hline & Média & $17,1 \mathrm{a}$ & $51,8 \mathrm{a}$ & $33,9 \mathrm{~b}$ \\
\hline \multirow[t]{5}{*}{ B } & Cocais & $15,7 \mathrm{~b}$ & $52,3 \mathrm{a}$ & $31,2 \mathrm{~d}$ \\
\hline & Guanhães & $15,3 \mathrm{~b}$ & 52,9 a & $40,1 \mathrm{~b}$ \\
\hline & Rio Doce & $17,3 \mathrm{a}$ & $50,5 \mathrm{~b}$ & 44,3 a \\
\hline & Santa Bárbara & $15,1 \mathrm{~b}$ & $52,4 \mathrm{a}$ & $38,2 \mathrm{c}$ \\
\hline & Média & $15,9 \mathrm{~d}$ & 52,0 a & 38,5 a \\
\hline \multirow[t]{5}{*}{$\mathrm{C}$} & Cocais & $15,2 \mathrm{a}$ & $50,8 \mathrm{~b}$ & 28,0 a \\
\hline & Guanhães & $15,7 \mathrm{a}$ & $51,4 \mathrm{a}$ & 28,1 a \\
\hline & Rio Doce & $16,1 \mathrm{a}$ & $51,5 \mathrm{a}$ & $26,0 \mathrm{~b}$ \\
\hline & Santa Bárbara & $14,6 \mathrm{a}$ & 51,9 a & 29,1 a \\
\hline & Média & $15,4 \mathrm{~d}$ & $51,4 \mathrm{~b}$ & $27,8 \mathrm{c}$ \\
\hline \multirow[t]{5}{*}{$\bar{D}$} & Cocais & $15,7 \mathrm{~b}$ & $51,2 \mathrm{a}$ & $31,2 \mathrm{c}$ \\
\hline & Guanhães & $17,0 \mathrm{a}$ & 50,7 a & 35,8 a \\
\hline & Rio Doce & $17,1 \mathrm{a}$ & $50,7 \mathrm{a}$ & $33,8 \mathrm{~b}$ \\
\hline & Santa Bárbara & $16,0 \mathrm{~b}$ & 51,2 a & 36,4 a \\
\hline & Média & $16,5 \mathrm{~b}$ & $51,0 \mathrm{c}$ & $34,3 \mathrm{~b}$ \\
\hline \multirow[t]{5}{*}{$\mathrm{E}$} & Cocais & $16,7 \mathrm{~b}$ & $51,0 \mathrm{~b}$ & 39,3 a \\
\hline & Guanhães & $17,4 \mathrm{a}$ & 51,9 a & 41,1 a \\
\hline & Rio Doce & $17,2 \mathrm{a}$ & $51,0 \mathrm{~b}$ & $31,6 \mathrm{~b}$ \\
\hline & Santa Bárbara & $16,4 \mathrm{~b}$ & $51,2 \mathrm{~b}$ & 40,2 a \\
\hline & Média & 16,9 a & $51,3 \mathrm{~b}$ & 38,1 a \\
\hline \multirow[t]{5}{*}{$\mathrm{F}$} & Cocais & $16,9 \mathrm{a}$ & $50,3 \mathrm{~b}$ & $38,3 \mathrm{~b}$ \\
\hline & Guanhães & $16,4 \mathrm{~b}$ & $50,9 \mathrm{~b}$ & 39,3 a \\
\hline & Rio Doce & $15,5 \mathrm{~b}$ & $52,2 \mathrm{a}$ & $36,7 \mathrm{~b}$ \\
\hline & Santa Bárbara & $16,5 \mathrm{a}$ & $50,6 \mathrm{~b}$ & 40,5 a \\
\hline & Média & $16,3 \mathrm{c}$ & $51,0 \mathrm{c}$ & 38,7 a \\
\hline
\end{tabular}

Médias seguidas de mesma letra, dentro de clone, não diferem entre si pelo teste de Scott-Knott a 5 \% de probabilidade.

Tabela 9- Valores médios dos efeitos de local para as características de polpação.

Table 9 - Average values of the site effects for the pulping characteristics.

\begin{tabular}{lccc}
\hline Local & kappa & RD $(\%)$ & Viscosidade $(\mathrm{cP})$ \\
\hline Cocais & $16,41 \mathrm{a}$ & $51,20 \mathrm{~b}$ & $33,00 \mathrm{c}$ \\
Guanhães & $16,41 \mathrm{a}$ & $51,59 \mathrm{a}$ & $37,08 \mathrm{a}$ \\
Rio Doce & $16,58 \mathrm{a}$ & $51,30 \mathrm{~b}$ & $34,00 \mathrm{~b}$ \\
Santa Bárbara & $15,91 \mathrm{~b}$ & $51,47 \mathrm{a}$ & $36,61 \mathrm{a}$ \\
\hline
\end{tabular}

\section{CONCLUSÕES}

- A densidade da madeira medida no DAP foi ligeiramente superior à da árvore inteira (cavacos). A regional de Santa Bárbara foi a que apresentou os menores valores de densidade básica da madeira, estando relacionada à menor espessura da parede das fibras.
- O efeito de clone, local e interação apresentaram efeito significativo na densidade básica determinada nos cavacos, dimensões das fibras, exceto a largura da fibra, e composição química, exceto o teor de extrativos. Na maioria dos casos, o efeito de clone foi mais intenso que o local (regional).

- As madeiras da região de Cocais se destacaram pelos seus maiores e menores teores de celulose e hemiceluloses, respectivamente. Esse resultado é explicado pelo fato de as árvores de regiões montanhosas possuírem, usualmente, maior quantidade de madeira de tração.

- As madeiras da regional de Santa Bárbara apresentam os menores e maiores teores de celulose e hemiceluloses, respectivamente.

R. Árvore, Viçosa-MG, v.33, n.6, p.1175-1185, 2009 
- O efeito de clone, local e interação foram significativos em todas as características da polpação.

- O clone F, plantado na região de Cocais e Santa Bárbara, foram considerados como de mais baixa atratividade para a produção de celulose, em virtude de sua baixa densidade básica, composição química desfavorável e baixo rendimento depurado de polpação.

- Os melhores rendimentos de polpação kraft foram alcançados com o clone B nas regiões de Guanhães $(53,8 \%)$ e Santa Bárbara $(53,4 \%)$, associado a baixos teores de lignina $(28,6 \%$ e $28,8 \%)$.

- Tendo-se em vista que o objetivo da polpação é a remoção seletiva da lignina e liberação da porção fibrosa da madeira, as madeiras com baixos teores de lignina e de extrativos propiciam melhor desempenho da polpação em termos de rendimento

\section{AGRADECIMENTOS}

À Fundação de Apoio à Pesquisa do Estado de Minas Gerais (FAPEMIG), pelo apoio financeiro; e à CENIBRA (Celulose Nipo Brasileira S.A.), pela imprescindível contribuição.

\section{REFERÊNCIAS}

BROWNING, B. L. The chemistry of wood. New York: John Wiley \& Sons, 1963. $689 \mathrm{p}$.

COSTA, M. M.; MOUTEER, A. H.; COLODETTE, J. L. Ácidos hexenurônicos- Parte I: Origem, quantificação, reatividade e comportamento durante a polpação Kraft. O Papel, v. 62, p.75-85, 2001.

FOELKEL, C. E.; MORA, E.; MENOCHELLI, S. Densidade básica: sua verdadeira utilidade como índice de qualidade da madeira de eucalipto para produção de celulose. In: CONGRESSO FLORESTAL BRASILEIRO, 6 , 1990, Campos do Jordão. Anais... São Paulo: 1990. p.719-128.

GOLDSCHIMID, O. Ultraviolet spectra. In: SARKANEN, K. V.; LUDWIG, C. H. Lignins: occurrence, formation, structure and reactions. New York: John Wiley \& Sons, 1971. p.241-298.

GOMIDE, J. L.; DEMUNER, B. J. Determinação do teor de lignina na madeira: método Klason modificado. O Papel, v.47, n.1, p.36-38, 1986.

R. Árvore, Viçosa-MG, v.33, n.6, p.1175-1185, 2009
GOMIDE, J. L. et al. Caracterização tecnológica, para produção de celulose, da nova geração de clone de Eucalyptus do Brasil. Revista Árvore, v.29, n.1, p.129-137, 2005.

KAAR, W. E. et al. The complete analysis of wood polysaccharides using HPLC. Journal of Wood Chemistry and Technology, v.11, n.4, p.447-463, 1991.

MALAN, F. A. Eucalyptus improvement for lumber production. In: SEMINÁRIO INTERNACIONAL DE UTILIZAÇÃO DA MADEIRA DE E EUCALIPTO PARA SERRARIA, 1995, São Paulo. Anais... São Paulo: IPEF/IPT, 1995. p.1-19.

RAMALHO, R. S. O uso de macerado no estudo anatômico de madeiras. Viçosa, MG: Universidade Federal de Viçosa, 1987. 4p.

SCAN. Scan Test Standard of Scandinavian Pulp, Paper and Board. Stockholm: 1994.

SCOTT, R. W. Colometric determination of hexenuronic acids in plant materials.

Analytical Chemistry, v.51, n.7, p.936-941, 1979.

SOLAR, R.; KACIK, F.; MELCER, I. Simple semimicro method for the determination of O-acetyl groups in wood and related materials. Nordic Pulp and Paper Research Journal, v.4, p.139-141, 1987

SOUZA, A. P.; DELLA LUCIA, R. M.; RESENDE, G. C. Estudo da densidade básica da madeira de Eucalyptus microcorys F. Muell, cultivado na região de Dionísio, MG. Revista Árvore, v.3, n.1, p.16-27, 1979.

TECHNICAL ASSOCIATION OF THE PULP AND PAPER INDUSTRY - TAPPI. Standard methods of technical association of the pulp and paper industry. Atlanta: 2002.

TOMAZELLO FILHO, M. Estrutura anatômica da madeira de oito espécies de Eucalyptus no Brasil. Revista IPEF, n.29, p.25-346, 1995. 
TIMELL, T. E. The chemical composition of tension wood. New York: 1969. p.173-181.

WALLIS, A.; WERNE, R.; WRIGHT, P. Chemical analysis of polysaccharides in plantation eucalypt woods and pulps. Appita Journal, v.49, p.427$432,1996$.
WEHR, T. R.; BARRICHELO, L. E.

G. Cozimento kraft com madeiras de Eucalyptus grandis de diferentes densidades básicas e dimensões de cavacos. In: CONGRESSO ANUAL DE CELULOSE E PAPEL, 25, São Paulo, 1992. Anais...São Paulo: ABTCP, 1992, p.161-177. v.25. p.1-17 
